\title{
Gerebral Reactions during Intrauterine Asphyxia in the Sheep. II. Evoked Electroencephalogram Responses
}

\author{
A. Hrbek, K. Karlsson, I. Kjellmer, ${ }^{[18]}$ T. Olsson, and M. Riha \\ Department of Pediatrics, the Department of Physiology, and the Department of Gynecology and Obstetrics I, University of \\ Göteborg, and the Research Laboratory of Medical Electronics, Chalmers University of Technolcgy, Göteborg, Sweden
}

\section{Extract}

Somatosensory evoked electroencephalogram (EEG) responses (SER) were recorded in 19 fetal lambs with gestational ages varying from 66 to 140 days. The ewes were anesthetized with chloralose and the fetuses were exteriorized with intact umbilical circulation. Periods of hypoxia at different $\mathrm{pH}$ levels were induced by ventilating the ewe with gas mixtures including $8-15 \%$ oxygen, either alone or combined with a $10 \% \mathrm{CO}_{2}$ mixture, or with an intravenous infusion of bicarbonate to the fetus.

The reaction of the SER during hypoxia followed two main patterns, one seen in the immature fetus and one in the more mature fetus. In the immature type of response (seen in all fetuses at or less than 110 days of gestational age) the primary response of the SER is more sensitive to oxygen lack than the secondary components, whereas in the mature type of response (seen at or more than 115 days of gestational age) the reverse is true.

At moderate degrees of hypoxia, $\mathrm{Pa}_{\mathrm{O}_{2}}$ between 15 and $20 \mathrm{~mm} \mathrm{Hg}$, the SER were moderately affected provided that the $\mathrm{pH}$ of the blood was within normal limits. At lower $\mathrm{Pa}_{\mathrm{o}}$, values, and when moderate hypoxia was combined with acidosis, the SER were seriously affected or completely abolished. At $\mathrm{Pa}_{\mathrm{o}}$, values above $20 \mathrm{~mm} \mathrm{Hg}$ an acidosis had no effect on the SER.

\section{Speculation}

The function of the fetal brain is seriously jeopardized by the combination of hypoxia and acidosis. Therefore, both hypoxia and acidosis should be counteracted during asphyxia to prevent brain damage.

\section{Introduction}

In certain conditions one of the few methods available for studies of brain function is that of evoked EEG responses (ER). Some promising results have already been presented. For example, Brierley et $a l$. and associates $[3,9]$ found in rhesus monkeys with experimental profound arterial hypotension that somatosensory ER changes correlated closely with the degree of brain damage and the severity of the metabolic deficit.

The same authors demonstrated the superiority of ER recordings over recordings of spontaneous EEG for the evaluation of the functional state of the brain in some situations which involve brain damage.

Using dogs, Lee et al. [8] elicited a consistent and gradual increase in latency and amplitude of certain 
components of visual ER by a decrease of serum sodium, chloride, and osmolality levels.

The influence of hypoxia and anoxia on ER has been studied in different animal species (cat, monkey). In all experiments the primary component of the ER exhibited the greatest resistance to anoxia, whereas the later components were more vulnerable $[1,2,11]$.

It must be emphasized that all the animals studied in the above investigations were adult. Our experiences with human neonates during hypoxia (idiopathic respiratory distress syndrome) and after asphyxia yielded the opposite result: the primary components of visual as well as of somatosensory ER were less resistant to hypoxia than the secondary components [5].

Evoked responses develop pre- and postnatally in all species in a characteristic manner. The development of somatosensory ER in fetal lambs was described by Molliver [10]. As in other species, there is increasing complexity and decreasing latency of the response with increasing maturity.

The present study was undertaken to use the ER technique in the fetal lamb in order to test whether this aspect of brain function would be affected at a certain reproducible degree of hypoxia, and whether other environmental influences, such as acidosis, would modify the cerebral response to hypoxia.

\section{Methods}

The experiments were conducted on 15 ewes of mixed breed with 19 fetuses ranging from 66 to 140 days of estimated gestational age. Complete experiments with both blood gas determinations and recordings of somatosensory evoked responses were obtained on 15 fetuses, between 94 and 140 days of gestational age. The gestational age was estimated from fetal weight and crown-rump length using standard curves [6].

The ewes were anesthetized with pentothal $(5 \mathrm{mg} / \mathrm{kg}$ body wt) followed by chloralose ( $30 \mathrm{mg} / \mathrm{kg}$ body $\mathrm{wt}$ ). The ewes were tracheotomized and ventilated with known gas mixtures, using an open circuit respirator. Maternal blood pressure was recorded through a catheter in the medial plantar artery of one foreleg. The same catheter was used for arterial blood sampling. The fetus was delivered onto a heated small table with intact umbilical circulation. Fetal arterial blood pressure and heart rate were recorded from a catheter in the right brachial artery. The same catheter was used for blood sampling.

Periods of hypoxia were induced by ventilating the ewe with gas mixtures with $8-15 \%$ oxygen. Different
$\mathrm{pH}$ levels were achieved by adding extra $\mathrm{CO}_{2}$ to the gas mixture or by giving an intravenous infusion of bicarbonate to the fetus.

A more detailed description of the preparation and the procedures is given in the accompanying paper [7].

Evoked EEG responses were recorded from the surface of the intact scalp. Cup electrodes were fixed over the part corresponding to the suprasylvian gyrus. The indifferent electrode was fixed at the posterior part of the head. At the beginning of the experimental series it was confirmed that the responses recorded from the scalp were similar to those recorded directly from the exposed cortex. The procedure of registration from the intact head precluded the damage to the brain that might otherwise occur in the course of the experiments, with a duration of several hours. The EEG was monitored on a Grass polygraph model $7 B$ [13], with an ink recorder. The data were also stored on a Philips FM tape recorder [14]. For averaging, a PDP 12 digital computer [15] was used. Electric stimulation was carried out with a battery-operated stimulator, with lead stimulation electrodes, placed $5 \mathrm{~mm}$ apart on the muzzle close to the nostrils. The stimulus was a rectangular pulse of 1-ms duration and of about $10 \mathrm{~V}$.

\section{Results}

The maturity of the fetal lamb determines the shape, the complexity, and the latency period of the SER. Figure 1 shows a few representative examples of the development of the SER at increasing degrees of maturity. It should be noted that the complete response

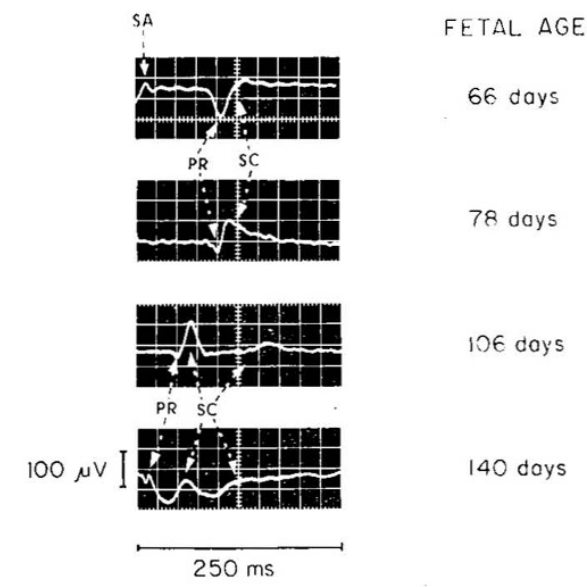

Fig. 1. The development of somatosensory evoked clectrocephalogram responses in fetal lambs from 66 to 140 days of gestational age. Primary responses $(P R)$ and secondary components $(S C)$ are marked. $S A$ : stimulation artifact. 
covers a long time period, so that a recording time of 250-500 ms is often necessary to register the complete set of slow, secondary components. As is apparent from Figure 1 increasing maturity leads to a shorter latency period and an increased complexity, with a higher frequency pattern of the response.

The different components of the SER were separated according to several criteria, one of the most important being the localization. The primary, specific response was in every experiment most distinctly recorded in the corresponding ipsilateral projection area, and in several instances present in the ipsilateral area exclusively. The secondary, nonspecific components were recorded from both hemispheres.

Hypoxia affected the SER profoundly when the oxygen tension of the fetal arterial blood was reduced below $20 \mathrm{~mm} \mathrm{Hg}$. The first reaction of the SER was a decrease in amplitude. In the most severe hypoxia, the whole SER was abolished. When the hypoxia was discontinued the SER returned gradually during the following 30-60 $\mathrm{min}$, except for those fetuses in which circulatory collapse occurred during the hypoxic period.

Hypoxia also affected the spontaneous EEG pattern recorded. However, this will be left out of the analysis since in several cases, particularly in the more immature fetuses, distinct SER continued to be recorded even though no spontaneous electrical activity, or only an amorphously irregular activity, was observed. Furthermore, it was repeatedly observed that, in cases with a distinct spontaneous electrical activity, this was preserved long after the SER demonstrated marked alterations.

The reaction of the SER during hypoxia followed two main patterns, one seen in the immature fetus and one in the more mature fetus.

An example of the response of the immature fetus is shown in Figure 2, left panel. The primary part of the SER proves to be more sensitive to lack of oxygen and decreases in amplitude and vanishes earlier than the secondary components. When, after the complete response is abolished, the hypoxia is discontinued, the
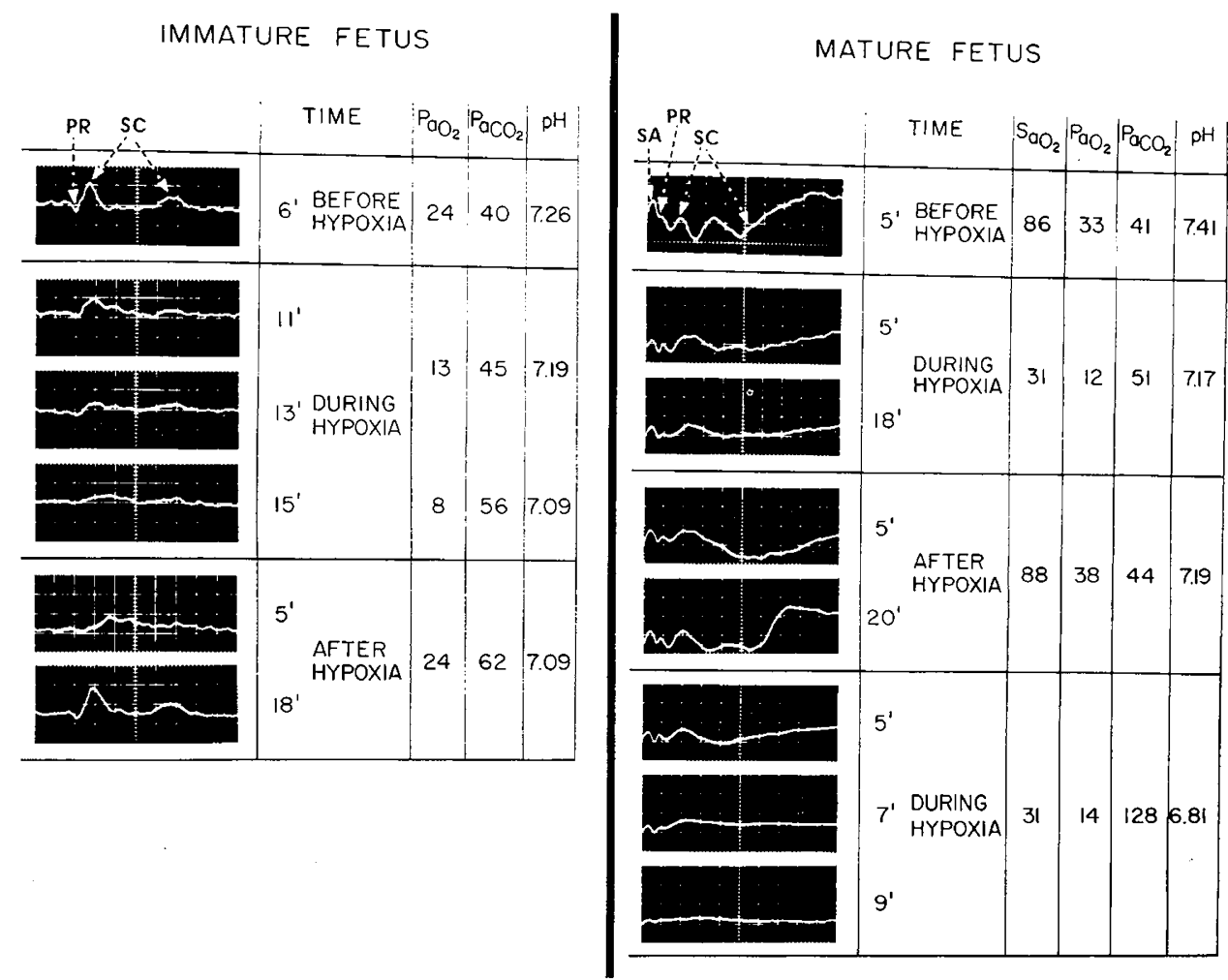

Fig. 2. The reactions of somatosersory evoked electroencephalogram responses (SER) to hypoxia. The left panel shows from an immature fetal lamb with an estimated gestational age of 106 days. Note that the primary response $(P R)$ is more sensitive to hypoxia than the secondary components $(S C)$. The right panel shows the reactions to two periods of hypoxia of the SER from a mature fetal lamb with an estimated gestational age of 125 days. Note that in this example the primary response is more resistant to hypoxia than the secondary complex. Calibration is as in Figure 1. In the responses from the mature fetus a stimulation artifact $(S A)$ is present. 
components of the SER return in the reverse order: first the secondary components (at $5 \mathrm{~min}$ ) and later the primary response (at $18 \mathrm{~min}$ ).

In the mature lamb fetuses the reverse type of response was observed. Figure 2, right panel, gives an example of a fetus of 125 days of gestational age. Here the primary response is more resistant to hypoxia and is affected first when the secondary components have already been extinguished. When the hypoxia is relieved the primary response reappears earlier than the secondary components.

In 14 experiments, the hypoxia was severe enough to extinguish the complete SER. In these experiments the sensitivity of the different components of the response was compared. Figure 3 gives a schematic comparison between the sensitivity of the primary and the secondary components to hypoxia for different degrees of maturity. Note that all instances at or under 110 days of gestational age demonstrate the immature type of response, whereas fetuses over 115 days show the mature response.

To grade the influence of hypoxia on the SER, all responses were classified in terms of the decrease, from the control state of the amplitude of the most sensitive component.

At moderate degrees of hypoxia, at $\mathrm{Pa}_{\mathrm{O}_{2}} \quad 15-20 \mathrm{~mm}$ $\mathrm{Hg}$, the SER were moderately affected provided the $\mathrm{pH}$ of the blood was within normal limits. At lower $\mathrm{PO}_{2}$ values, or when moderate hypoxia was combined with acidosis, the SER were seriously affected or completely abolished. Figure 4 is a graphic representation of the relation between $\mathrm{Po}_{2}, \mathrm{pH}$ and the $\mathrm{SER}$. At $\mathrm{Pa}_{\mathrm{O}_{2}}$ values above $20 \mathrm{~mm} \mathrm{Hg}$, acidosis had no effect on the SER.

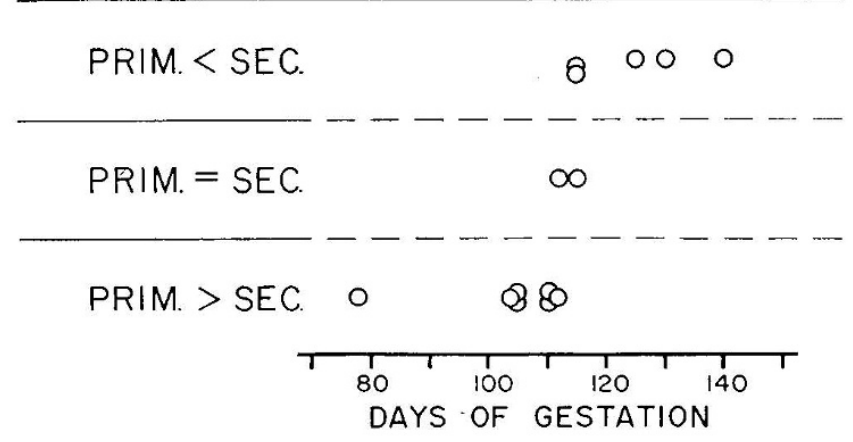

Fig. 3. A graphic demonstration of the relation between fetal age and the sensitivity to hypoxia of primary versus secondary complex of somatosensory evoked electroencephalogram responses. At a low gestational age the primary (PRIM.) response is more sensitive than the secondary (SEC.) response.

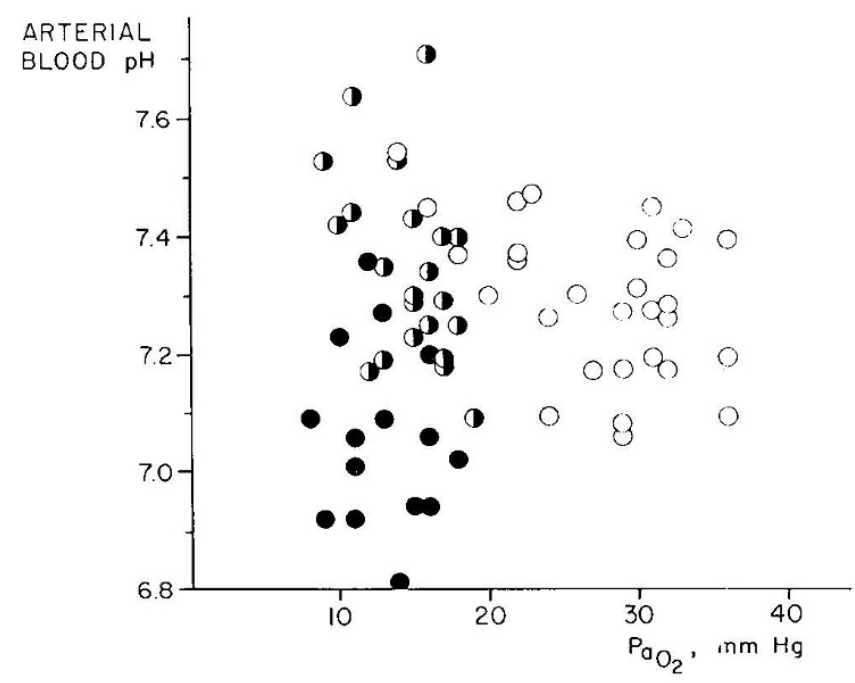

Fig. 4. The effect of varying arterial $\mathrm{pH}$ at different levels of hypoxia on somatosensory evoked electrocncephalogram responses (SER). O: SER not affected; 1 : SER distinctly affected (amplitude decreased to more than $50 \%$ of control); : SER completely extinguished.

\section{Discussion}

The development of SER in the fetal lamb during maturation has been described in detail by Molliver [10]. Our results demonstrate a close resemblance to his (Fig. I), although the present results were obtained via scalp electrodes, fixed to the skin, and not by the direct cortical recording technique. Our results contain more information about the late components than Molliver's, since we used the longer recording period of 250 ms.

Our results corroborate earlier views of the advantage of using ER over spontaneous EEG [3, 9] in studies of this type. This is particularly true in the immature fetuses where spontaneous electric activity from the brain is sparse or absent.

The marked vulnerability of the primary component of the SER in comparison with secondary components in the immature fetus (Figs. 2 and 3) has not been described previously, but corresponds to the pattern seen in the preterm and full term human baby [5]. At 110115 days of gestational age, corresponding to some $75 \%$ of the total fetal period, the response turns into the mature type (Figs. 2 and 3) described previously in adult animals of different species $[1,2,11]$. This finding is in agreement with the view of the newborn lamb as a neurologically mature creature.

The evolution of changes in the SER at increasing degrees of hypoxia follows a reproducible pattern. At normal or increased $\mathrm{pH}$ levels, the SER is unaffected 
down to a $\mathrm{PO}_{2}$ in the arterial blood of $20 \mathrm{~mm} \mathrm{Hg}$, i.e. the lower limit of normality (Table I, Reference 7). Below this limit the SER are distinctly altered with a reduction of the amplitude to below $50 \%$ of the control value. When the $\mathrm{Pa}_{2}$ is reduced below a level of about $12-15 \mathrm{~mm} \mathrm{Hg}$ the SER are completely abolished. If acidosis is added to the hypoxia the SER are affected at higher $\mathrm{PO}_{2}$ values and are already extinguished at $\mathrm{Pa}_{\mathrm{O} .2}$ as high as $18 \mathrm{~mm} \mathrm{Hg}$ (Fig. 4). At normal oxygen tensions, on the other hand, a change of $\mathrm{pH}$ between 7.4 and 7.06 does not provoke any changes of the SER.

If the influence of hypoxia on the SER depends on the amount of oxygen delivered to the brain, rather than on the partial pressure of oxygen in the tissue, the combined effect of hypoxia and acidosis might be due to the shift of the hemoglobin dissociation curve with $\mathrm{pH}$, the Bohr effect. The mature fetus in Figure 2 demonstrates the magnitude of the Bohr effect in one specific case. During the first period of hypoxia $\mathrm{pH}$ was only moderately reduced and the $\mathrm{Po}_{2}$ of $12 \mathrm{~mm} \mathrm{Hg}$ corresponded to a saturation of $31 \%$. In the second hypoxic period an acidosis was added and exactly the same oxygen saturation was achieved, but now at a $\mathrm{PO}_{2}$ of $14 \mathrm{~mm} \mathrm{Hg}$. Thus, the Bohr effect contributes to a moderate extent only in explaining the detrimental effect on the SER of the combination of hypoxia and acidosis. Instead, it appears reasonable to combine this item of information with the knowledge gained from studies on the metabolic rate of the brain in lamb fetuses [7]. Here, it was demonstrated that the oxygen consumption of the brain was reduced significantly more when hypoxia was combined with an acidosis than when hypoxia occurred at normal $\mathrm{pH}$ levels. Thus, the combination of acidosis and hypoxia might be deleterious to the electric activity of the brain by interfering with the energy metabolism. This view is supported by the findings in rats that the energy state of the tissue, "the energy charge potential," is reduced at significantly higher oxygen tensions when ischemic hypoxia is combined with a respiratory acidosis than when the ischemia is induced at normal pH levels [4].

The present findings are also of interest in the light of the report of Windle et al. [12] that the newborn monkey develops less morphologic signs of brain damage when experimental asphyxia is combined with alkalizing treatment than when acidosis is allowed to develop in connection with the hypoxia.

Thus, various experimental series using different animal species and different techniques to evaluate the impact of asphyxia on the brain appear unanimous on one point; the combination of hypoxia and acidosis is more dangerous for the brain than hypoxia alone.

\section{Summary}

In an attempt to analyze factors responsible for the brain damage during intrauterine asphyxia, SER were studied in the fetal lamb to test whether this aspect of cerebral function would be affected at a certain reproducible degree of hypoxia and whether other environmental influences, such as acidosis, would modify the cerebral response to hypoxia.

Nineteen fetal lambs, ranging from 66 to 140 days of gestational age, were studied in the acute, exteriorized preparation. Hypoxia was induced by ventilating the ewe with appropriate gas mixtures. Acidosis or alkalosis were produced by a $\mathrm{CO}_{2}$-rich gas mixture to the ewe or by an infusion of alkali into the fetus.

The SER of immature lamb fetuses (less than 110 days of gestational age) responded to hypoxia in the same manner as the SER from human neonates; the primary response of the SER complex was more sensitive to lack of oxygen than were the secondary components. More mature lamb fetuses (over 115 days of gestational age) demonstrated the opposite response pattern, with the primary response more resistant to hypoxia than the secondary components, as is the case for adult animals of various species.

When $\mathrm{Pa}_{\mathrm{O}_{2}}$ was diminished at normal or high $\mathrm{pH}$ levels, the SER were affected below $20 \mathrm{~mm} \mathrm{Hg}$ but were abolished first at severe degrees of hypoxia. When an acidosis was superimposed, however, the SER were abolished at even moderate degrees of hypoxia.

\section{References and Notes}

1. Baumgartner, G., Creutzfeldt, O., and Jung, R.: Microphysiology of cortical neurons in acute anoxia and in retinal ischemia. In: H. Gastaut and J. S. Meyer: Cerebral Anoxia and the Electroencephalogram, pp. 5-34 (Charles C Thomas, Publisher, Springfield, Ill., 1961).

2. Bostem, F., Lesevre, N., and Naquet, R. L.: Potentiels évoqués chez le singe Papio papio: Effets de l'anoxie. Electromyography, 8: 263 (1968).

3. Brierley, J. B., Brown, A. W., Excell, B. J., And Meldrum, B. S.: Brain damage in the rhesus monkey resulting from profound arterial hypotension. I. Its nature, distribution and general physiological correlates. Brain Res., 13: 68 (1969).

4. Ekiöf, B., MacMillan, V., and Siesjö, B. K.: The effect of ischemia upon the energy state of the brain. In: C. Fieschi: Ccrebral Blood Flow and Intracranial Pressure, p. 60 (S. Karger, Basel, 1972).

5. Hrbek, A., Karlberg, P., KJell.mer, I., and Olsson, T.: Evoked EEG responses in newborns with asphyxia and IRDS. Pediat. Res., 6: 6l (1972). 
6. Jouberi, D. M.: A study of prenatal growth and development in the sheep. J. Agr. Sci., 47: 382 (1956).

7. KJellmer, I., Karlsson, K., Olsson, T., and Rosén, K. G.: Cerebral reactions during intrauterine asphyxia in the sheep. I. Circulation and oxygen consumption in the fetal brain. Pediat. Res., 7: 50 (1974).

8. LeE, S. I., Klass, D. W., and Wakim, K. G.: Changes in visual evoked potentials induced by electrolyte alterations in dogs. Electroencephalogr. Clin. Neurophysiol., 27: 677 (1969).

9. Meldrum, B. S., And Brierley, J. B.: Brain damage in the rhesus monkey resulting from profound arterial hypotension. II. Changes in the spontaneous and evoked electrical activity of the neccortex. Brain Res., 13: 101 (1969).

10. Molliver, M. E.: An ontogenetic study of evoked somesthetic cortical responses in the sheep. Progr. Brain Res., 26 (Developmental Neurology): (1967).

11. Naquet, R. L., and Fernandez-Guardirola, A.: Effects of various types of anoxia on spontaneous and evoked cerebral activity in the cat. In: H. Gastaut and J. S. Meyer: Cerebral
Anoxia and the Electroencephalogram, pp. 72-88 (Charles C Thomas, Springfield, Ill., 1961).

12. Windle, W. F., Jacobson, H. N., Ramirez de Arellano, M. J. R., AND Combs, C. M.: Structural and functional sequelae of asphyxia neonatorum in monkeys (Macaca mulatta). Res. Publ. Ass. Res. Nerv. Ment. Dis., 39: 169 (1962).

13. Grass Instrument Co., Quincy, Mass.

14. Philips Electronics Instruments, Mt. Vernon, N. Y.

15. PDP, Digital Equipment Corp., Maynard, Mass.

16. Dr. A. Hrbek is the recipient of a Fellowship in Pediatric Research, The Fels Research Institute for the Study of Human Development, Yellow Springs, Ohio.

17. This research was supported by Grants nos. 19X-2591, 19X3949, and 19V-2939, Swedish National Research Council, and National Association against Heart and Lung Disease, Swedish Board for Technical Development, Förstamajblomman.

18. Requests for reprints should be addressed to: I. KJELLMER, M.D., Department of Pediatrics, Ostra Sjukhuset, Göteborg, Sweden.

19. Accepted for publication September 12, 1973. 